PROCEEDINGS OF THE

AMERICAN MATHEMATICAL SOCIETY

Volume 131, Number 4, Pages 1011-1014

S 0002-9939(02)06616-9

Article electronically published on July 17, 2002

\title{
FINITENESS OF REPRESENTATION DIMENSION
}

\author{
OSAMU IYAMA \\ (Communicated by Martin Lorenz)
}

\begin{abstract}
We will show that any module over an artin algebra is a direct summand of some module whose endomorphism ring is quasi-hereditary. As a conclusion, any artin algebra has a finite representation dimension.
\end{abstract}

M. Auslander introduced a concept of representation dimension of artin algebras in $[\mathrm{A}]$, which was a trial to give a reasonable way of measuring homologically how far an artin algebra is from being of finite representation type ([X1], [FGR]). His methods given there have been effectively applied not only for the representation theory of artin algebras [ARS, but also for the theory of quasi-hereditary algebras of Cline-Parshall-Scott [CPS] by Dlab-Ringel in DR2. Unfortunately, much seems to be unknown about representation dimension itself. In particular, Reiten asked in 1998 whether any artin algebra has a finite representation dimension or not (cf. $\S 2.3(2)$ ). In this paper, we will give a positive answer to this question ( $\$ 1.2)$ by showing that any module is a direct summand of some module whose endomorphism ring is quasi-hereditary $(\S 1.1)$. Our method is to construct a certain chain of subcategories of $\bmod \Lambda(\S 2.2)$, which was applied to solve Solomon's second conjecture on zeta functions of orders in [3]. We will formulate it in terms of rejective subcategories (§2.1), which was effectively applied in [I1] to study the representation theory of orders and give a characterization of their finite Auslander-Reiten quivers in [12.

Note. After the author submitted this paper, Professor Xi kindly informed him that Theorem 1.1 and Corollary 1.2 were stated in [X2] as conjectures, where the former was given by Ringel and Yamagata. He thanks Professor Xi and Professor Yamagata for valuable comments.

1.

In this paper, any module is assumed to be a left module. For an artin algebra $\Lambda$ over $R$, let $\bmod \Lambda$ be the category of finitely generated left $\Lambda$-modules, $J_{\Lambda}$ the Jacobson radical of $\Lambda$, dom.dim $\Lambda$ the dominant dimension of $\Lambda$ [T], $\mathrm{I}_{\Lambda}(X)$ the injective hull of the $\Lambda$-module $X$ and ()$^{*}:=\operatorname{Hom}_{R}\left(, \mathrm{I}_{R}\left(R / J_{R}\right)\right): \bmod \Lambda \leftrightarrow$ $\bmod \Lambda^{o p}$ the duality. For $X \in \bmod \Lambda$, we denote by add $X$ the full subcategory of $\bmod \Lambda$ consisting of direct summands of a finite direct sum of $X$. The representation dimension of $\Lambda$ is defined by rep.dim $\Lambda:=\inf \{\operatorname{gl} \cdot \operatorname{dim} \Gamma \mid \Gamma \in A(\Lambda)\}$, where $A(\Lambda)$ is the collection of all artin algebras $\Gamma$ such that $\operatorname{dom} \cdot \operatorname{dim} \Gamma \geq 2$ and $\operatorname{End}_{\Gamma}\left(\mathrm{I}_{\Gamma}(\Gamma)\right)$ is

Received by the editors August 6, 2001 and, in revised form, October 29, 2001.

2000 Mathematics Subject Classification. Primary 16G10; Secondary 16E10.

(C)2002 American Mathematical Society 
Morita-equivalent to $\Lambda$. Then rep.dim $\Lambda=\inf \left\{\operatorname{gl} \cdot \operatorname{dim} \operatorname{End}_{\Lambda}(M) \mid M \in \bmod \Lambda\right.$ such that $\Lambda \oplus \Lambda^{*} \in$ add $M$ \} holds by [A].

1.1. Theorem. Let $\Lambda$ be an artin algebra. Then any $M \in \bmod \Lambda$ is a direct summand of some $N \in \bmod \Lambda$ such that $\operatorname{End}_{\Lambda}(N)$ is a quasi-hereditary algebra.

1.2. Corollary. Let $\Lambda$ be an artin algebra. Then rep.dim $\Lambda$ has a finite value which is not greater than $2 l-2$, where $l$ is the length of $a\left(\Lambda, \operatorname{End}_{\Lambda}\left(\Lambda \oplus \Lambda^{*}\right)\right)$-module $\Lambda \oplus \Lambda^{*}$.

2 .

In the rest of this paper, any subcategory $\mathcal{C}^{\prime}$ of an additive category $\mathcal{C}$ is assumed to be full and closed under direct sums. Let $\mathcal{J}_{\mathcal{C}}$ be the Jacobson radical of $\mathcal{C}$ and $\left[\mathcal{C}^{\prime}\right]$ the ideal of $\mathcal{C}$ consisting of morphisms which factor through some object in $\mathcal{C}^{\prime}$. Thus $\mathcal{J}_{\mathcal{C}}(X, X)$ forms the Jacobson radical of the $\operatorname{ring} \mathcal{C}(X, X)$ for any $X \in \mathcal{C}$.

2.1. Let $\mathcal{C}$ be an additive category and $\mathcal{C}^{\prime}$ a subcategory of $\mathcal{C}$.

(1) $\mathcal{C}^{\prime}$ is called a right rejective subcategory of $\mathcal{C}$ if the inclusion functor $\mathcal{C}^{\prime} \rightarrow \mathcal{C}$ has a right adjoint $\mathbb{F}: \mathcal{C} \rightarrow \mathcal{C}^{\prime}$ with a counit $\epsilon[\mathrm{HS}]$ such that $\epsilon_{X}$ is a monomorphism for any $X \in \mathcal{C}$ (cf. [I1], 5.1). This is equivalent to that, for any $X \in \mathcal{C}$, there exists a monomorphism $g \in \mathcal{C}(Y, X)$ with $Y \in \mathcal{C}^{\prime}$ which induces an isomorphism $\mathcal{C}(, Y) \stackrel{g}{\longrightarrow}\left[\mathcal{C}^{\prime}\right](, X)$ on $\mathcal{C}$ (cf. [11, 5.2).

(2) $0=\mathcal{C}_{m} \subseteq \mathcal{C}_{m-1} \subseteq \cdots \subseteq \mathcal{C}_{0}=\mathcal{C}$ is called a right rejective chain if $\mathcal{J}_{\mathcal{C}_{n} /\left[\mathcal{C}_{n+1}\right]}=$ 0 holds and $\mathcal{C}_{n+1}$ is a right rejective subcategory of $\mathcal{C}_{n}$ for any $n(0 \leq n<m)$. In this case, if $\Gamma:=\mathcal{C}(M, M)$ is an artin algebra for an additive generator $M$ of $\mathcal{C}$, then $\Gamma$ is a quasi-hereditary algebra with a heredity chain $0=\left[\mathcal{C}_{m}\right](M, M) \subseteq$ $\left[\mathcal{C}_{m-1}\right](M, M) \subseteq \cdots \subseteq\left[\mathcal{C}_{0}\right](M, M)=\Gamma$.

Dually, we define a left rejective subcategory and a left rejective chain.

2.1.1. Let $\mathcal{C}^{\prime}$ be a right rejective subcategory of $\mathcal{C}$ and $\mathcal{C}^{\prime \prime}$ a subcategory of $\mathcal{C}^{\prime}$. Then $\mathcal{C}^{\prime} /\left[\mathcal{C}^{\prime \prime}\right]$ is a right rejective subcategory of $\mathcal{C} /\left[\mathcal{C}^{\prime \prime}\right]$ since the isomorphism $\mathcal{C}(, \mathbb{F}(X))$ $\stackrel{\epsilon_{X}}{\longrightarrow}\left[\mathcal{C}^{\prime}\right](, X)$ induces an isomorphism $\left[\mathcal{C}^{\prime \prime}\right](, \mathbb{F}(X)) \stackrel{\epsilon_{X}}{\longrightarrow}\left[\mathcal{C}^{\prime \prime}\right](, X)$. Moreover, if $\mathcal{C}^{\prime \prime}$ is a right rejective subcategory of $\mathcal{C}^{\prime}$, then it is a right rejective subcategory of $\mathcal{C}$.

2.1.2. Proof of 2.1(2). $\mathcal{C}_{m-1}$ is also a right rejective subcategory of $\mathcal{C}$ by 2.1.1. Let $\mathbb{F}$ be the right adjoint of the inclusion $\mathcal{C}_{m-1} \rightarrow \mathcal{C}$. Then $I:=\left[\mathcal{C}_{m-1}\right](M, M)$ is isomorphic to a projective $\Gamma$-module $\mathcal{C}(M, \mathbb{F}(M))$, and $I J_{\Gamma} I=0$ holds by $\mathcal{J}_{\mathcal{C}_{m-1}}=0$. Since $\left[\mathcal{C}_{m-1}\right]^{2}=\left[\mathcal{C}_{m-1}\right]$ holds, $I$ is a heredity ideal of $\Gamma$. Since $0=\mathcal{C}_{m-1} /\left[\mathcal{C}_{m-1}\right] \subseteq$ $\mathcal{C}_{m-2} /\left[\mathcal{C}_{m-1}\right] \subseteq \cdots \subseteq \mathcal{C}_{0} /\left[\mathcal{C}_{m-1}\right]=\mathcal{C} /\left[\mathcal{C}_{m-1}\right]$ is again a right rejective chain by 2.1.1, we obtain the assertion inductively.

2.2. Our results 1.1 and 1.2 immediately follow from the following lemma (put $M:=\Lambda \oplus \Lambda^{*}$ for 1.2$)$.

Lemma. Let $\Lambda$ be an artin algebra and $M \in \bmod \Lambda$. Put $M_{0}:=M, M_{n+1}:=$

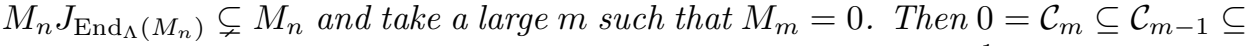
$\cdots \subseteq \mathcal{C}_{0}=\mathcal{C}$ gives a right rejective chain for $\mathcal{C}_{n}:=\operatorname{add} \bigoplus_{l=n}^{m-1} M_{l}$. Thus $\Gamma:=$ $\operatorname{End}_{\Lambda}(N)$ is a quasi-hereditary algebra for $N:=\bigoplus_{l=0}^{m-1} M_{l}$ such that gl.dim $\Gamma \leq$ $2 m-2$. 
Proof. (i) Note that there exists a surjection $f_{n, l} \in \operatorname{Hom}_{\Lambda}\left(\bigoplus M_{n}, M_{l}\right)$ for any $n<l$.

(ii) Define a functor $\mathbb{F}_{n}: \bmod \Lambda \rightarrow \bmod \Lambda$ by

$$
\mathbb{F}_{n}(X):=\sum_{Y \in \mathcal{C}_{n},} \sum_{f \in \mathcal{J}_{\bmod \Lambda}(Y, X)} f(Y) .
$$

Then a natural transformation $\epsilon: \mathbb{F}_{n} \rightarrow 1$ is defined by the inclusion $\epsilon_{X}: \mathbb{F}_{n}(X) \rightarrow$ $X$. By (i), $\mathbb{F}_{n}\left(M_{n}\right)=M_{n} J_{\operatorname{End}_{\Lambda}\left(M_{n}\right)}=M_{n+1} \in \mathcal{C}_{n+1}$ holds. Thus $\mathcal{J}_{\mathcal{C}_{n}}(, X)=$ $\left[\mathcal{C}_{n+1}\right](, X)=\mathcal{C}_{n}\left(, \mathbb{F}_{n}(X)\right) \epsilon_{X}$ holds on $\mathcal{C}_{n}$ for any indecomposable $X \in \mathcal{C}_{n}-\mathcal{C}_{n+1}$.

(iii) Fix indecomposable $X \in \mathcal{C}_{n}$. Put $Y:=\mathbb{F}_{n}(X)$ and $g:=\epsilon_{X}$ if $X \notin \mathcal{C}_{n+1}$, and $Y:=X$ and $g:=1_{X}$ if $X \in \mathcal{C}_{n+1}$. By (ii), $Y \in \mathcal{C}_{n+1}$ and $\mathcal{C}_{n}(, Y) \stackrel{g}{\longrightarrow}\left[\mathcal{C}_{n+1}\right](, X)$ is an isomorphism on $\mathcal{C}_{n}$. Thus $\mathcal{C}_{n+1}$ is a right rejective subcategory of $\mathcal{C}_{n}$. Since $\mathcal{J}_{\mathcal{C}_{n} /\left[\mathcal{C}_{n+1}\right]}=0$ holds by (ii), our chain is right rejective. Now gl.dim $\Gamma \leq 2 m-2$ follows from [DR1].

2.3. Remark. (1) The dual version of 2.2 is the following lemma, which gives a variation of the theorem of Auslander and Dlab-Ringel in [A] and DR2 by putting $M:=\Lambda$.

Lemma. Let $\Lambda$ be an artin algebra and $M \in \bmod \Lambda$. Put $M_{0}:=M, M_{n+1}:=$ $M_{n} /\left\{x \in M_{n} \mid x J_{\operatorname{End}_{\Lambda}\left(M_{n}\right)}=0\right\}$ and take a large $m$ such that $M_{m}=0$. Then $0=\mathcal{C}_{m} \subseteq \mathcal{C}_{m-1} \subseteq \cdots \subseteq \mathcal{C}_{0}=\mathcal{C}$ gives a left rejective chain for $\mathcal{C}_{n}:=\operatorname{add} \bigoplus_{l=n}^{m-1} M_{l}$.

Thus $\Gamma:=\operatorname{End}_{\Lambda}(N)$ is a quasi-hereditary algebra for $N:=\bigoplus_{l=0}^{m-1} M_{l}$ such that gl.dim $\Gamma \leq 2 m-2$.

(2) By a result of Igusa-Todorov (【T, 0.8), rep.dim $\Lambda \leq 3$ implies fin.dim $\Lambda<\infty$. Thus, from the viewpoint of the finitistic global dimension conjecture, it is an interesting question whether any artin algebra $\Lambda$ satisfies rep.dim $\Lambda \leq 3$ or not $\mathrm{A}$ ].

\section{REFERENCES}

[A] M. Auslander: Representation dimension of Artin algebras, Lecture notes, Queen Mary College, London, 1971.

[ARS] M. Auslander, I. Reiten, S. O. Smale: Representation theory of Artin algebras. Cambridge Studies in Advanced Mathematics, 36, Cambridge University Press, Cambridge, 1995. MR 96c:16015. MR 98e:16011

[CPS] E. Cline, B. Parshall, L. Scott: Finite-dimensional algebras and highest weight categories. J. Reine Angew. Math. 391 (1988), 85-99. MR 90d:18005

[DR1] V. Dlab, C. M. Ringel: Quasi-hereditary algebras. Illinois J. Math. 33 (1989), no. 2, 280291. MR 90e: 16023

[DR2] V. Dlab, C. M. Ringel: Every semiprimary ring is the endomorphism ring of a projective module over a quasihereditary ring. Proc. Amer. Math. Soc. 107 (1989), no. 1, 1-5. MR 89m:16033

[FGR] R. M. Fossum, P. Griffith, I. Reiten: Trivial extensions of abelian categories. Lecture Notes in Mathematics, Vol. 456, Springer-Verlag, Berlin-New York, 1975. MR 52:10810

[HS] P. J. Hilton, U. Stammbach: A course in homological algebra. Graduate Texts in Mathematics, 4. Springer-Verlag, New York, 1997, xii+364 pp. MR 97k:18001

[I1] O. Iyama: $\tau$-categories II: Nakayama pairs and rejective subcategories, to appear in Algebras and Representation theory.

[I2] O. Iyama: $\tau$-categories III: Auslander orders and Auslander-Reiten quivers, to appear in Algebras and Representation theory.

[I3] O. Iyama: A proof of Solomon's second conjecture on local zeta functions of orders, to appear in J. Algebra.

[IT] K. Igusa, G. Todorov: On the finitistic global dimension conjecture, preprint.

[T] H. Tachikawa: Quasi-Frobenius rings and generalizations. Lecture Notes in Mathematics, Vol. 351, Springer-Verlag, Berlin-New York, 1973. MR 50:2233 
[X1] C. C. Xi: On the representation dimension of finite dimensional algebras. J. Algebra 226 (2000), no. 1, 332-346. MR 2001d:16027

[X2] C. C. Xi: Representation dimension and quasi-hereditary algebras, to appear in Adv. Math.

Department of Mathematics, Kyoto University, Kyoto, 606-8502, Japan

E-mail address: iyama@kusm.kyoto-u.ac.jp

Current address: Department of Mathematics, Himeji Institute of Technology, Himeji, 6712201, Japan

E-mail address: iyama@sci.himeji-tech.ac.jp 